\title{
Load Identification of Different Halbach-Array Topologies on Permanent Magnet Synchronous Generators Using the Coupled Field-Circuit FE Methodology
}

\author{
Pedram Asef ${ }^{1} *$, Ramon B. Perpina ${ }^{2,}$ M. Reza Barzegaran $*^{3}$, Andrew Lapthorn ${ }^{4}$, and Daniela Mewes ${ }^{5}$ \\ ${ }^{, I}$ Electrical Engineering Department, Polytechnic University of Catalonia-BarcelonaTech, Barcelona 08019, Spain \\ ${ }^{2}$ Electrical Engineering Department, Polytechnic University of Catalonia-BarcelonaTech, Barcelona 08019, Spain \\ ${ }^{3}$ Phillip M. Drayer Dept. of Electrical Engineering, Lamar University, Beaumont, USA \\ ${ }^{4}$ Electrical and Computer School, University Of Canterbury, Christchurch, New Zealand \\ ${ }^{5}$ Industrial Engineering and Management, KTH Royal Institute of Technology, Lindstedsvagen, Sweden
}

\section{Article history:}

Received

Received in revised form

Accepted

Available online

Keywords:

Full rectifier

Electric Machine Design

Finite Element Analysis

Load optimization

Gap consideration

\begin{abstract}
In this paper, the influence of gap consideration on load identification under various Halbacharray-based topologies (HABOs) is investigated while the system is on-duty. The load characteristics of a radial flux generator with closed-slots and the exterior rotor topology is discussed, where the effect of eddy-currents have been observed. This comparative study deals with the consideration of the combined moment of inertia calculation that demonstrates how electromagnetic-based post processing calculations are estimated without the aid of nominal machine parameter values. The analysis was performed using a 2-D finite-element simulation of different HABOs with the gap consideration between the segments. Additionally, a comprehensive comparison with no gap was considered. Also, the dynamic analysis using an uncontrolled conventional rectifier model was used to derive effected key output parameters such as torque, output power, power factor, and line-to-line voltage. The major objective of the study is to determine corresponding load results in order to employ the most suitable and capable magnetization topology from the load perspective in the PM synchronous generator (PMSGs). Accordingly, the maximum power (MP) point was carried out to maximize the output DC power. With respect to the combined moment of inertia estimation, the load parameter estimation was verified experimentally on a surface-mounted PMSG using different magnetization topologies. Furthermore, commercial and environmental issues of the project are considered to reduce $\mathrm{CO}_{2}$ emissions as part of green power generation development.
\end{abstract}

2017 Elsevier Ltd. All rights reserved.

\section{Introduction}

\subsection{Motivation}

Distributed generation and electric vehicles normally employ permanent magnet synchronous generators (PMSGs) due to their high efficiency and power density characteristics, where the load is often supplied through a diode rectifier connected across the armature terminals. For this purpose, the electromagnetic (EM) and mechanical machine parameters should be accurately calculated. The most significant electromagnetic parameters are permanent magnet flux linkage, $d$-axis and q-axis inductances, line to line peak voltage, and rotor electromagnetic torque. The mechanical parameters are prevailing with respect to the load characteristics, which is preferred in real applications where the commercial machine drive has the capability to estimate these parameters online or offline. Accordingly, many investigations have been reported recently.

Basically, the estimation of the mechanical parameters requires the electromagnetic torque and rotor speed as the inputs to the estimation model [1]-[7]. In reality, the rotor speed can be

\footnotetext{
* Corresponding authors.

e-mails: pedram.asef@estudiant.upc.edu (Pedram Asef), mbarzegaran@lamar.edu (M. R. Barzegaran)
} 
measured directly by the position sensor, while the electromagnetic torque needs to be calculated via the torqueconstant and measured current. A typical solution is to use the nominal value of torque-constant of the generator to nearly calculate the electromagnetic torque [1].

Moreover, to reduce the design costs, the inverter can be replaced by a full-bridge diode rectifier followed by a DC-DC single switch converter [1]-[3] instead of using a pulse width modulation (PWM) converter acting as a controlled rectifier.

The main motivation is to take the gap consideration between each magnetic segment into account and evaluate its influence on the load side for different type of magnetization orientations.

\subsection{Literature review}

Lacchetti et. al [8], analyzes some operational issues of threephase PMSG connected to a diode rectifier. This configuration coupled to a single-switch DC-DC converter is used in small scale wind energy conversion systems, and in energy harvesting systems to reduce costs.

Weiming et. al [9], discusses an equivalent circuit model of a synchronous generator with a diode-bridge rectifier and backelectromotive force (EMF) load is presented in the paper. The principle of harmonic balance is applied, which makes it possible to construct a state-space mathematical model for the system. The criterion of stability is obtained for the system under small disturbances. Additionally, it is shown that the system's stability can effectively be improved by installing a short-circuited quadrature-axis winding on the machine rotor.

Wang et. al [10], the performance of an axial flux (PMSG) feeding an isolated rectifier load is studied using a coupled-circuit, time-stepping, 2-D finite-element analysis. The short- circuit performance is also investigated. The second-order serendipity quadrilateral elements are used in the analysis to obtain the accurate results of the analysis. Non-linearities in the field and electric circuit are taken into consideration. With the strong nonlinear diode parts existing in the external circuit, the special interpolating algorithm is used for the motion interface between the stator and rotor to overcome the non-convergence problem in the computation. The algorithm is validated by comparison with conventional algorithm for an AFPMSG feeding with a rectifier resistive load.

\subsection{Our contribution}

This paper challenges the mentioned electromagnetic and mechanical key designs of the machine via the coupled fieldcircuit SPMSG finite element (FE) models under a dynamic load analysis while the machine is feeding a diode rectifier load. Correspondingly, an evaluation will be conducted on the influence of the different HABOs on the following EM parameters " $L_{d}$ " axis inductance in $(\mathrm{mH})$, " $L_{q}$ " q-axis inductance $(\mathrm{mH})$, "VLL(pk)" the peak line to line voltage in $(\mathrm{V})$, in which the gap between each magnet segment is under consideration. In addition, various geometric models will be studied in the mechanical perspective of the design due to a different combined moment of inertia This comparative study is using an uncontrolled three-phase full rectifier load (Fig. 1) that has provided a comprehensive comparison through the load mechanical torque, its torque ripples, the output load power, the power factor, combined moment of inertia calculation by the given experimental layout. In where, the "blue" parameters will be calculated in the following sections. All other mentioned design parameters with and without the gap consideration are also traced.

Through the schematic (in Fig. 1), it should be mentioned that the constant speed via a prime mover has been adjusted to the rated speed of 150 (rpm) coupled with the cross-sectional SPMSG. Also, the three output phases are measured for the power factor at the voltage.ampere/ power factor (VA/PF). Therefore, the coupled field-circuit SPMSG finite element (FE) time-stepped model under the dynamic analysis will be performed differently and reported in Section IV. The following section will discuss the analytical issues in the study along with notable considerations and assumptions. Following this, the section of comparison and investigation will represent the comparative results through a comprehensive discussion. Finally, the conclusion will summarize the highlights and outcomes of the research. The conclusion as the last section ends up with a overview of the work with outcomes. Also, the electromagnetic performance of the novel models will be verified numerically and experimentally.

\section{Analytical statement of the study}

This section can be divided into four parts in order to introduce the considerations expressions such as the gap and combined moment of inertia, the uncontrolled rectifier three-phase and the corresponding coupled used system's methodology.

\section{A. The Gap Consideration of PMSG}

This consideration must be taken into account due to the different geometrical and EM highlights involved, unless an inaccurate design approximation exists. Therefore, this study considered the gap between each magnetic segment dipped in EPOXY-KLEBER 8601, as demonstrated in Fig. 2.

In $\mathrm{HABO}$ each magnetic block has a specific magnetization orientation [11], where the orientation of each block can be given as

$$
\theta_{m, i}=\theta_{i}(1+p)
$$

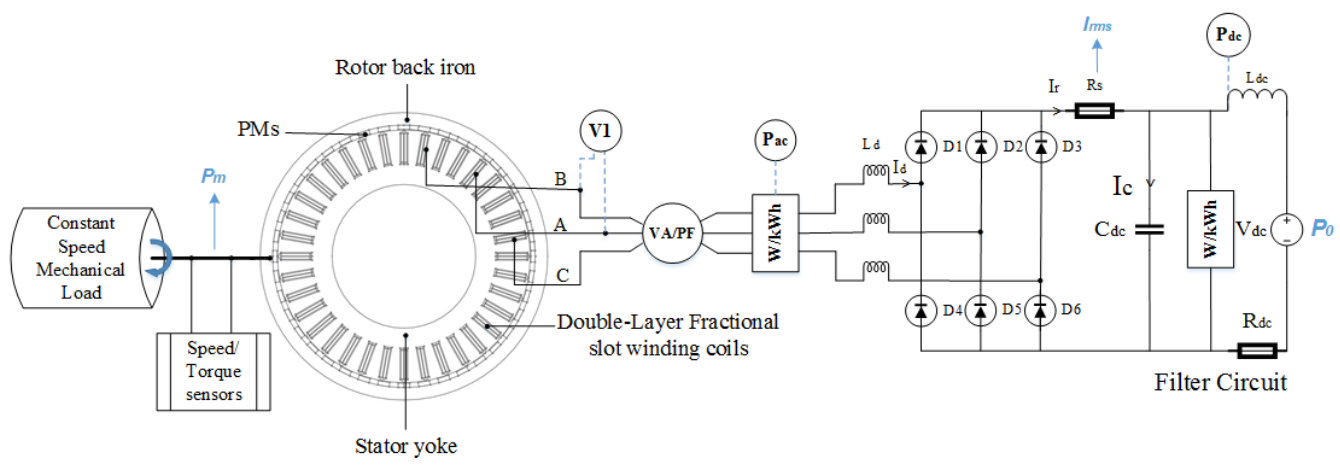

Fig. 1. The experimental setup of the coupled field-circuit PMSG finite element model feeding an uncontrolled three-phase full rectifier load 
here $\theta_{i}$ shows the angle between the center of the ith segment under condition of zero angle of orientation $(\theta=0)$. Consequently [11], the $\theta_{i}$ can be redefined as given

$$
\theta_{i=1,2,3, \ldots, 2 p l}=\frac{(i-1) \pi}{p . l}
$$

where $p, l$ are the pole pair number and the segment number per pole.

The HABO consists of three pieces. "side-magnet" "midmagnet" and "end side-magnet". Each piece will be magnetized in parallel orientation but with a different angle of orientation. Therefore, the ratio of the nth PM pole-arc to the pole-pitch can be expressed [11] as follows

$$
R_{p a / p p}=W_{m} / W_{p}
$$

where $\mathrm{Wm}$ and $\mathrm{Wp}$ are the pole-arc of mid-magnet and the pole-pitch, respectively.

The gap consideration as shown [11] in Fig. 2 can define the distance between magnets as presented by $A_{l}$, and $A_{2}$. Thus, the ratio of $A_{1}$, and $A_{2}$ (as the distance between PMs, and the width segment) has been defined as the relative pole arc coefficient

$$
\alpha_{p a}=A_{2} / A_{1}
$$

Accordingly, the gap between PM segments in this paper has been calculated by the relative pole-arc coefficient and the radius at the center of the PM [7], given as

$$
\Delta A=A_{2}-A_{1}=\left(1-\alpha_{p a}\right) \cdot \frac{\pi\left(R_{0}+R_{1}\right)}{2 p . l}
$$

where $R_{0}$, and $R_{l}$ are the inner and outer radius of the magnets in $(\mathrm{mm})$. In the majority of papers, the consideration of gap between segments is neglected by setting $\alpha_{p a}$ to one [11].

\section{B. The Combined Moment of Inertia of PMSG Models}

The combined moment of inertia (also known as combined angular mass) is a mechanical design parameter that has to be calculated accurately. It is necessary for the load identification which determines the required torque for a desired angular acceleration about a rotational axis. This depends on the body's mass distribution as well as the axis chosen, with larger moments needing more torque to transform the body's rotation. Basically, the moment of inertia relies on the radiuses, used material, and related density [12]. Therefore, the combined moment of inertia has been calculated based on Fig. 3 for each individual geometry and corresponding regions through the below equation

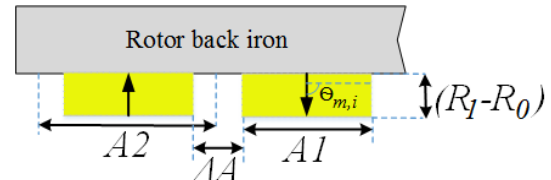

(a)

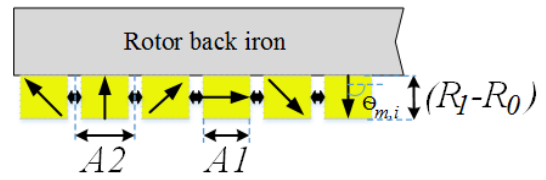

(d)

$$
\tilde{I}=\sum_{n=1}^{3}\left[\int_{Q} r^{2} d m\right]
$$

where $\tilde{I}$ is integrated over the entire mass $(Q)$ of the machine that consists of three regions of the SPMSG. The rotor back iron, the PMs, and the stator yoke are region 1,2, and 3, respectively. The conventional parallel topology (CPT), 2-segmented, modified-2segmented, and 4-segmented are 0.786, 0.8185, 0.8157, and $0.8146\left(\mathrm{~kg} \cdot \mathrm{m}^{2}\right)$, respectively.

TABLE I THE DENSITY OF USED MATERIALS

\begin{tabular}{c|ccccc}
\hline Parts & $\begin{array}{c}\text { Rotor } \\
\text { back iron }\end{array}$ & PMs & $\begin{array}{c}\text { EPOXY- } \\
\text { KLEBER } \\
8601\end{array}$ & $\begin{array}{c}\text { Stator } \\
\text { yoke }\end{array}$ & Coils \\
\hline Density & 7800 & 7600 & 1.31 & 7800 & 8890 \\
Unit & $\mathrm{kg} / \mathrm{m}^{\wedge} 3$ & $\mathrm{~kg} / \mathrm{m}^{\wedge} 3$ & $\mathrm{~g} / \mathrm{cm}^{\wedge} 3$ & $\mathrm{~kg} / \mathrm{m}^{\wedge} 3$ & $\mathrm{~kg} / \mathrm{m}^{\wedge} 3$ \\
\hline
\end{tabular}

\section{The Three-phase Uncontrolled full Rectifier Load Behavior}

Based on the steady-state analytical model that is developed in [8], and [17], the behavior of the rectifier at Fig. 1 can be studied through a constant voltage $\left(\mathrm{V}_{0}\right)$, and series of connected resistances and inductances as

$$
e_{k}(t)=\hat{V} \cdot \sin \left(\omega t-(k-1) \cdot \frac{2 \pi}{3}\right), k=1,2,3
$$

where $\hat{V}$ stands for the amplitude of the phase voltage and $\omega$ is the angular frequency. The diode forward voltage drop is $v_{D}$ due to the diode current $\left(i_{D}\right)$, which has been modelled via a threshold voltage $\left(V_{D}\right)$, and a resistance $\left(\mathrm{R}_{\mathrm{D}}\right)$

$$
v_{D}\left(i_{D}\right)=V_{D}+R_{D} i_{D}
$$

where $i_{D}>0$. Since the DC load caters an adequate inductance, the load DC current $\left(I_{d c}\right)$ will remain ripple-free and constant. The rectified voltage that is the yield of three-phase diode rectifier includes the generated ripples by the rectifier [13].

In this modelling system, two key parameters referred to as the $m$-ratio and $\varphi_{z}$ can be addressed as follows

$$
m=\frac{V_{0}+2 V_{D}}{E_{p k}}, \cos \left(\varphi_{z}\right)=\frac{R+R_{D}}{Z}
$$

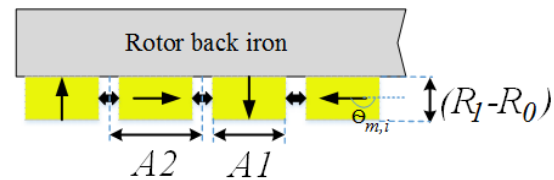

(b)

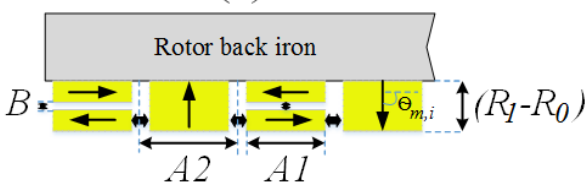

(c)

Fig. 2 The scheme of different EM orientation topologies with the gap consideration between each segment, a) conventional parallel topology (CPT), b) 2segmented unbalanced $\mathrm{HABO}, \mathrm{c}$ ) modified 2-segmented HABO with gap consideration between magnets, and d) 4-segmented HABO 


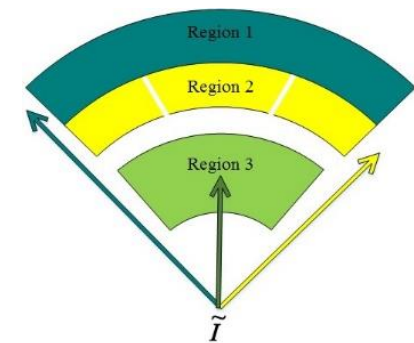

Fig. 3 The region definition with the related radiuses

where $Z$ stands for the modulus of the internal impedance that consists of $R_{D}$ which further consists of the incremental resistance of the diodes by

$$
Z=\sqrt{\left(R+R_{D}\right)^{2}+(\omega L)^{2}}
$$

The peak value of the internal $\operatorname{EMF}\left(E_{p k}\right)$ is

$$
E_{p k}=\omega k_{\omega} N \varphi
$$

where $k_{\omega}, N$, and $\varphi$ are the winding factor for the fundamental harmonic, the number of turns in series per phase, the peak magnet flux, and $\omega$ is the stator angular frequency. Additionally, the average DC current as well as the AC RMS current can be expressed as

$$
\begin{gathered}
I_{0}=\frac{E_{p k}}{Z} I_{0}^{*}\left(\theta_{x}, m, \varphi_{z}\right) \\
I_{r m s}=\frac{E_{p k}}{Z} I_{r m s}^{*}\left(\theta_{x}, m, \varphi_{z}\right)
\end{gathered}
$$

In Eq. (12-13), is $\theta_{x}$ an auxiliary variable angle that is integrated via an additional constraint equation which is

$$
F\left(\theta_{x}, m, \varphi_{z}\right)=0
$$

Essentially, Eq. (12-14) relies on the $m$ and $\varphi_{z}$ which define an assumption to ignore $\theta_{x}$, Fig. 4 demonstrates the different operation modes, in which, Fig. 4(a), (b), and (c) are representing $2 / 0,2 / 3$, and $3 / 3$ operations, respectively.

The power delivered to the DC bus can be defined as given

$$
P_{0}=\frac{E_{p k}^{2}}{Z}\left(m-\frac{2 V_{D}}{E_{p k}}\right) \cdot I_{0}^{*}\left(\theta_{x}, m, \varphi_{Z}\right)
$$

The three-phase full rectifier load coupled with the PMSG defines the efficiency through iron and mechanical losses lumped in $\left(P_{f e, \text { mech }}\right)$ which can be calculated as

$\eta=\frac{P_{0} / E_{p k}^{2} / z}{\left[P_{0} / E_{p k}^{2} / z\right]+\left[3 \cdot I_{r m s}^{* 2} \cos \phi z\right]+\left[2 I_{0}^{*}\left(V_{D} / E_{p k}\right)+\left[P_{f e, \text { mech. }}\left(Z / E_{p k}^{2}\right)\right]\right.}(16)$
The iron losses are approximated in $\left(P_{f e, \text { mech }}\right)$ are approximated [8] through the no-load iron losses, as well as also mechanical losses as given

$$
P_{\text {fe,mech } .}=\omega\left(c_{0}+c_{1} \omega\right)
$$

$c_{0}$, and $c_{l}$ are lumped the frictional, mechanical, and hysteresis losses and mostly take into account the eddy current losses.

\section{The Eddy Currents Effect}

According to [8], the important harmonic derived from the stator current necessitates that the eddy-current losses are taken into account. As resistance increases due to the eddy currents, a coefficient $\left(k_{R}>1\right)$ has been employed for the calculation of joule losses through increasing the windings DC resistance. Essentially, the $k_{R}$ factor is a function of the square of the frequency and normal to the slot leakage flux direction. Thus, the winding losses can be expressed as

$$
\left\{\begin{array}{c}
P_{w}=3 I_{r m s}^{2} \cdot k_{R} \cdot R \\
P_{w}=3 I_{r m s}^{2} \tilde{k}_{R} \cdot R \rightarrow \text { if }: I_{2}=\text { distorted }
\end{array}\right.
$$

where $I_{2}$, and $R$ are the stator winding current and the resistance of the winding. Additionally, when the current is distorted, a new resistance factor should be defined as follows

$$
\tilde{k}_{R}=1+\left(k_{R}-1\right) \sum_{k} k^{2}\left(\frac{I_{k}^{2}}{I_{r m s}^{2}}\right)
$$

$\tilde{k}_{R}$ factor is function of the defined key parameters $m$-ratio and $\varphi_{z}$, on the operation point of the rectifier system that is derived by the eddy-currents. The $k_{R}$ is based on the various characteristic angles of the impedance $Z\left(\varphi_{z}\right)$ that are $\pi / 2, \pi / 4$, and $\pi / 6$ results in the new resistance factors of 1.30986, 1.22891, and 1.19621 (rad). The lower $\varphi_{z}$ results in higher eddy-current losses due to the distorted current waveforms. It should be mentioned that fundamental $k_{R}$ has been bounded by 1.15 which indicates that through a proper division and transportation of the conductors in the stator slots, the contribution of eddy-current losses can be more than $23 \%$ due to the distortion [8], [18].

\section{Comparison and Investigation}

2-D FE analysis has been performed (via Fig. 5) on the different HABOs with the gap consideration between each magnet of the SPMSG-rectifier-load system (as shown in Fig. 1) with 33208, 32864,36140 , and 51069 nodes were used for the CPT, 2segmented, modified-2-segmented, and 4-segmented HABOs, were used for the respectively.

As a simplified outcome of the previous sections, a flowchart based on the proceedings of the paper has been presented as Fig. 6. This demonstrates how the entire study has been developed from
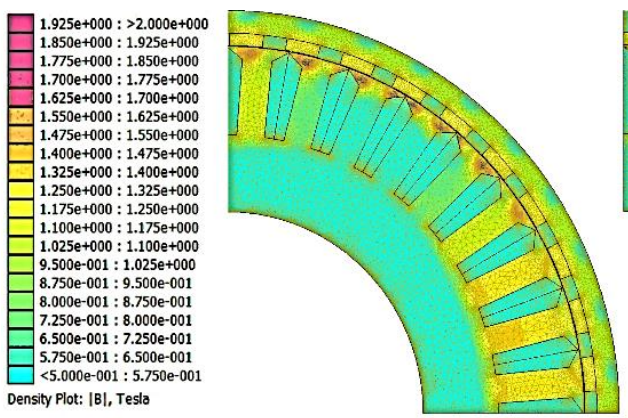

(a)

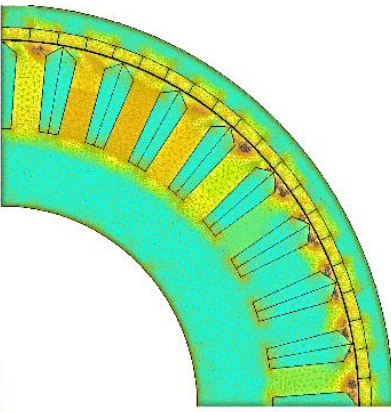

(b)

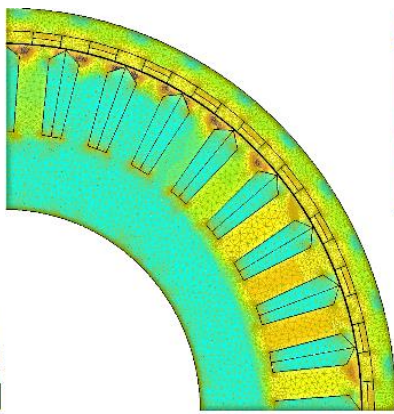

(c)

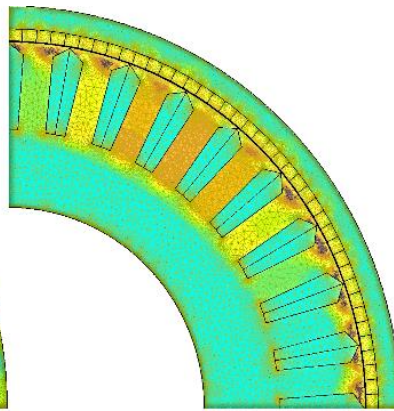

(d)

Fig. 5 The FE-field analysis including meshing with the gap consideration between each segment, a) the CPT, b) 2-segmented HABO, c) modified-2segmented HABO, and d) 4-segmented HABO 
the initial input design data (such as slot/ pole number, the pole thickness of the magnet, pole-width, slot type and dimension, stack length, air-gap length, and etc.) of each model which was then defined in the algorithm. Following this, the calculation of coordinate points and geometry modeling was performed by the LUA program with automatic handling of boundary conditions, required key parameters have been computed by the 2-D FEA solver. Additionally, the time-stepped (transient) analysis was performed using the FE field analysis data and the circuit data (including external and the rectifier load) [19] simultaneously in order to calculate the $\mathrm{P} 0$ and $\eta$.

The larger DQ-axis inductances are caused by the larger coercivity $(\mathrm{A} / \mathrm{m})$ produced by the magnets in each model. Thus, the DQ-plane inductances of each model has been presented in Fig. 7. Accordingly, the figure has mentioned that how the

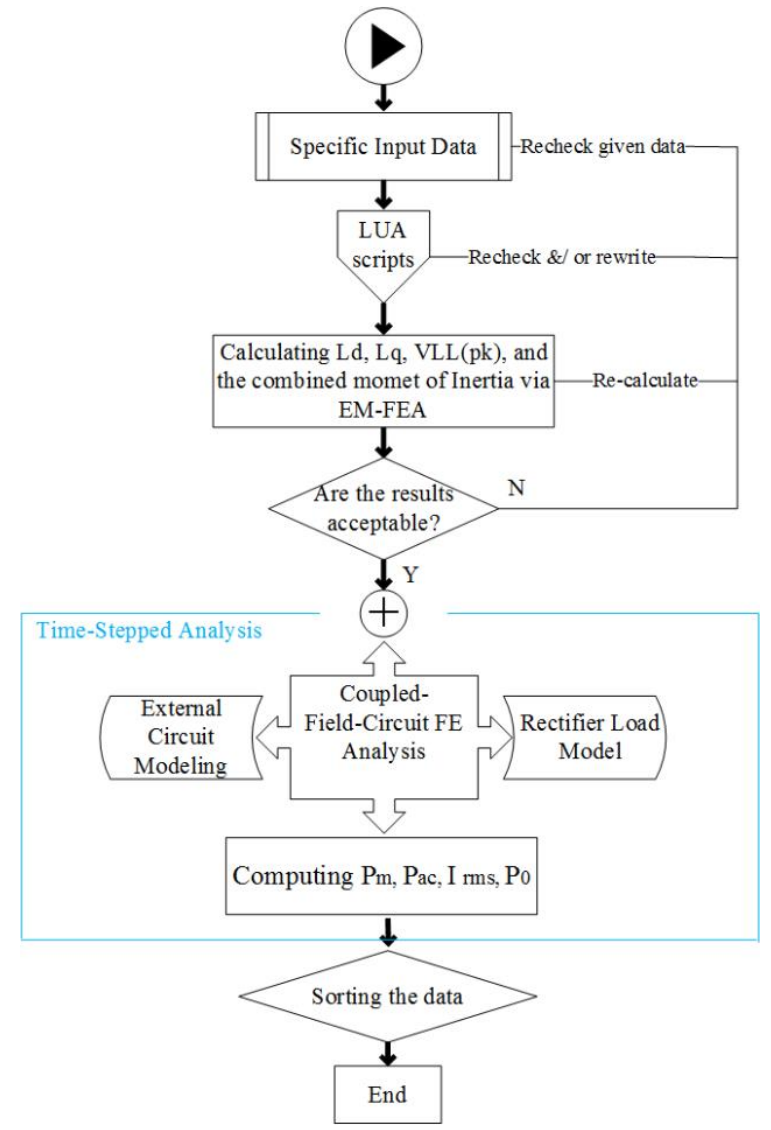

Fig. 6 The DQ-axis inductances FE computations via different models

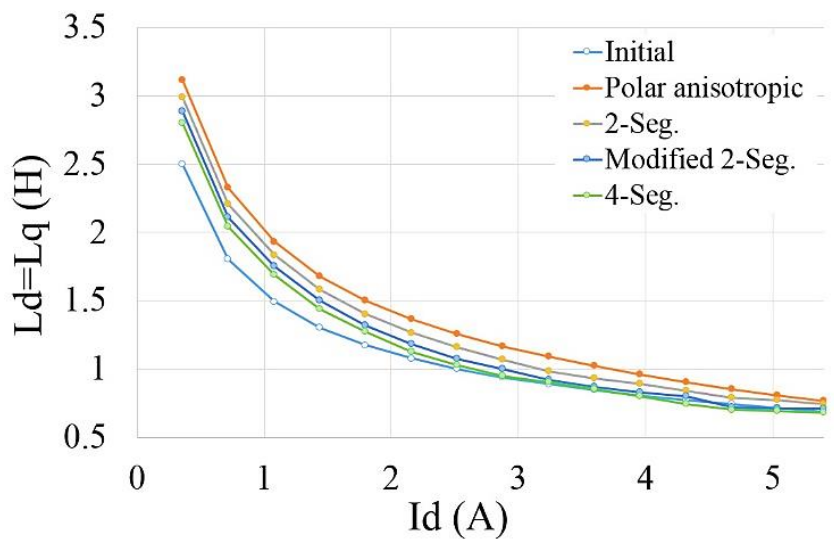

Fig. 7 The simplified procedure of the used methodology

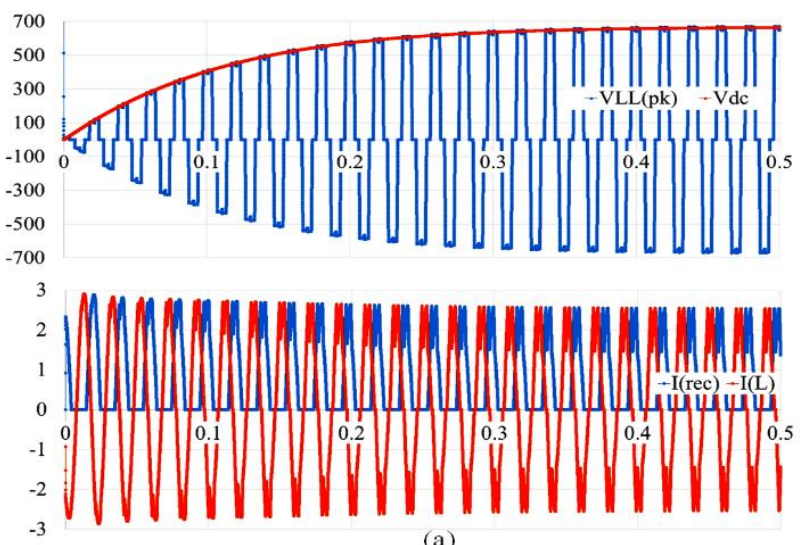

(a)
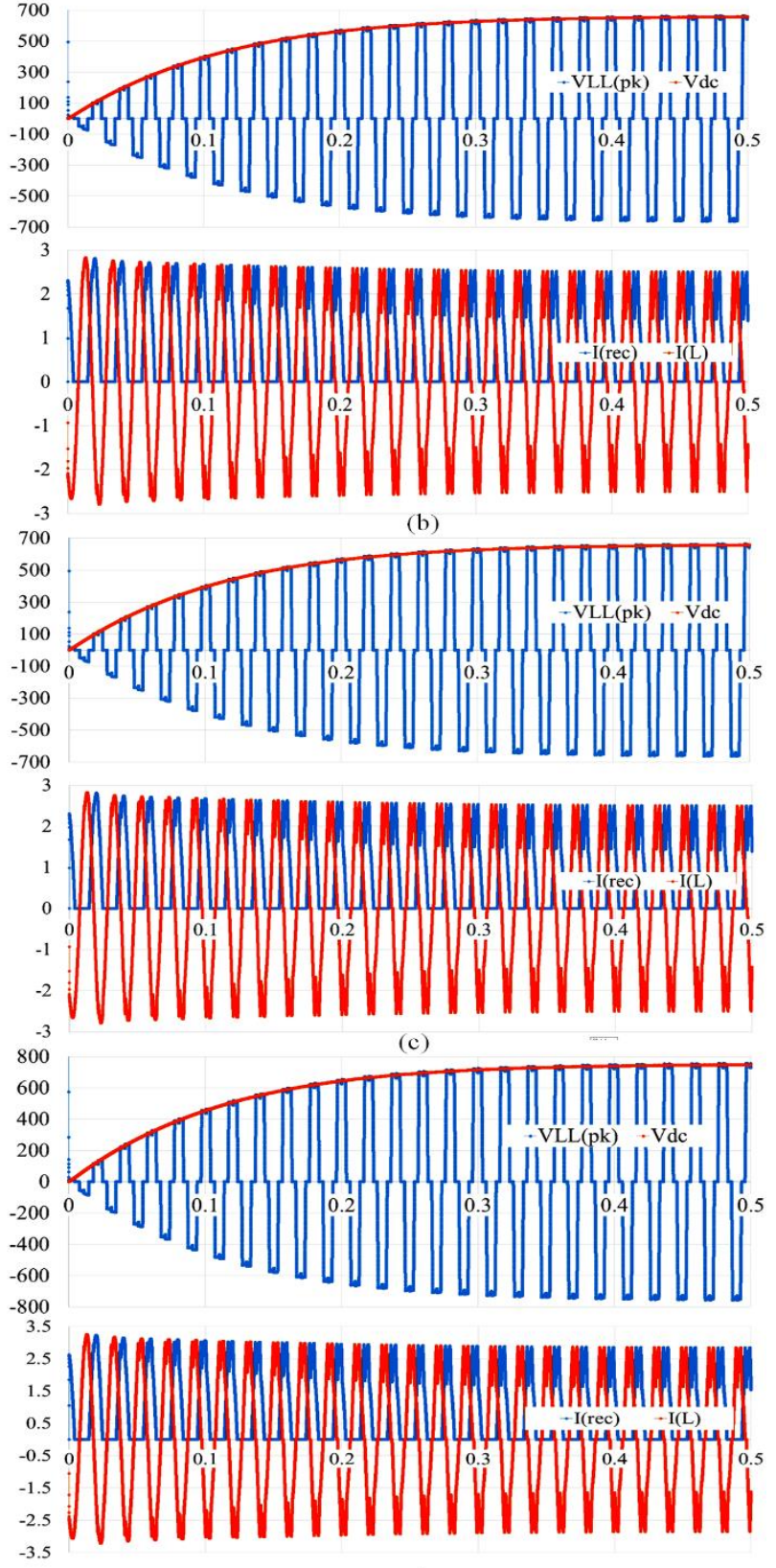

(d)

Fig. 8. The scheme of different HABO with the gap consideration between each segment, a) the CPT, b) the 2-segmented HABO, c) modified 2-segmented $\mathrm{HABO}$, and d) the 4-segmented HABO

For instance, due to an increased number of involved gaps (instead of high coercivity in comparison with other models), the 4-segmented model has been ranked with the lowest d-axis inductance among all the HABO models. Additionally, the polar 
anisotropic (continuous distribution) of the non-segmented model produced the greatest amount of inductances. The considered value of d-inductance of each model (included the CPT model) has been used at the nominal current of 2.12 (A). Fig. 8(a) represents the initial FE-CPT model connected to the three-phase rectifier load, which had a rectified current $\left(I_{r}\right)$ with average value of 0.6 (A). Additionally, for the 2-segmented HABO model shown in Fig. 8(b), there are considerable increases in VDC, and $I_{r}$. The modified-2-segmented HABO model seen by Fig. 8(c) that has improved the behavior of the classic-2-segmented model through maximizing VDC, $I_{r}$. However, the 4-segmented HABO model responded with the greatest maximization of the mentioned calculated parameters VDC, $I_{r}$.

The power factor $(\mathrm{PF})$ calculation deals with the complexity of the mechanical structure and the magnet consumption in the SPMSGs. Although the structure and the operation principle of the SPMSGs are different based on the magnet consumption and the related geometrical changes, the relationship between the electrical parameters (such as power factor) can still be investigated through the classical synchronous machine theory. Essentially, the PF is the ratio of the active and apparent power. If the fundamental harmonic components of the voltage and current are much higher than the higher harmonic components (low total harmonic distortion (THD) of the voltage and current), the PF has been calculated simply as the cosine of the displacement between the voltage and current (phase angle) [20-21] which can be written as

$$
P F(N I)=\cos \left(\arctan \left(\frac{Q}{P}\right)\right)=\frac{P}{P_{s}}
$$

where $P, Q$, and $P_{s}$ (shown through Fig. 9) are the active, reactive, and apparent powers, respectively. The accurate calculated power factors of the studied models are all 0.95 which represents that the width/pole pitch ratio, and the length/pole pitch ratio are the same in both the CPT and HABO-based models. However, the achieved MP for different model has been seen through Fig. 9, 10, and 11.

The Fig. 10(a) represents that the maximum reachable output DC power at a current of 0.854 (A), and $P F=0.95$. Fig. 10(b) presents that the maximum $P_{0}(I, \cos \varphi)$ of the 2 -segmented HABO model as function of the current and the PF will be achievable at the current of 1.037 (A). In Fig. 10(c), the maximum $P_{0}(I, \cos \varphi)$ of the modified-2-segmented HABO can be generated at the current of 1.038 (A), and $\mathrm{PF}=0.95$. In Fig. 10(d), the 4-segmented HABO model with maximum $P_{0}(I, \cos \varphi)$ can be generated at a current of 1.107 (A), and $\mathrm{PF}=0.95$. In addition, the FE timestepped behavior of the power $P_{0}(I, \cos \varphi)$ can be seen in via Fig. 10 , in which the studied models of 4-segmented, modified-2segmented, 2-segmented HABO models, and the CPT model are

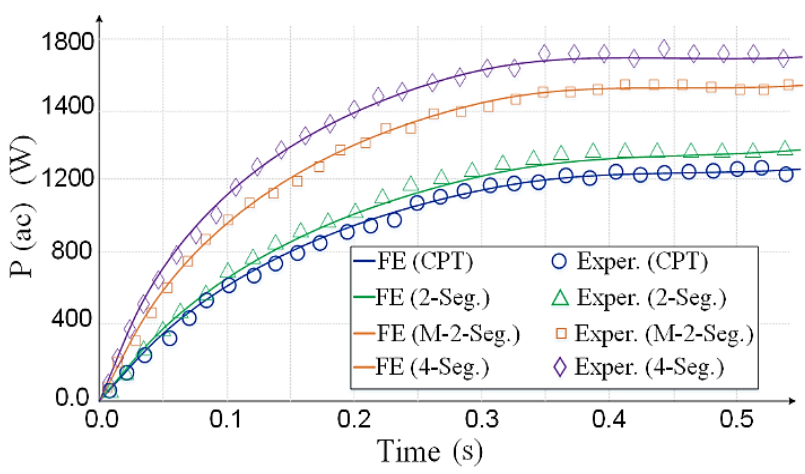

Fig. 9. Theoretical and experimental $P_{a c}$ comparison with gap consideration

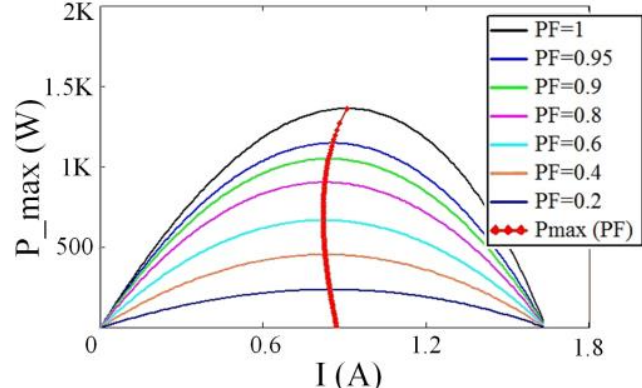

(a)

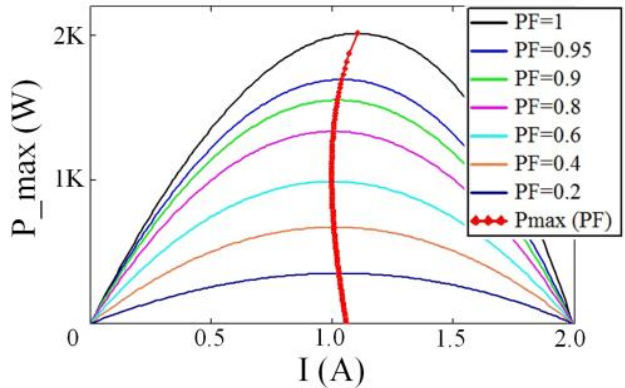

(b)

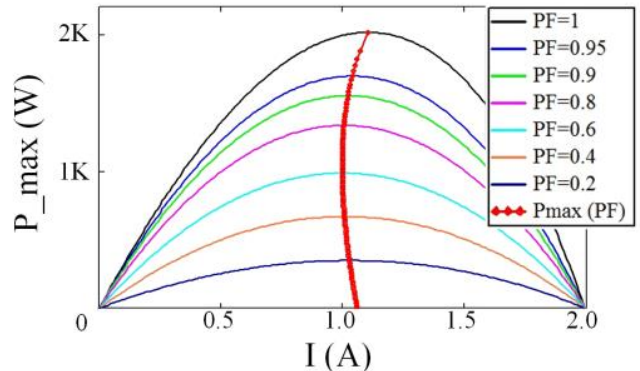

(c)

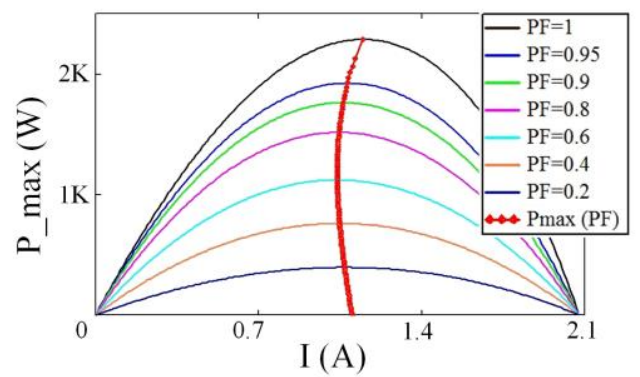

(d)

Fig. 10. The maximum output power of different EM orientation topologies with the gap consideration between each segment, a) the CPT, b) the 2segmented $\mathrm{HABO}$, c) modified 2-segmented HABO, and d) the 4-segmented $\mathrm{HABO}$

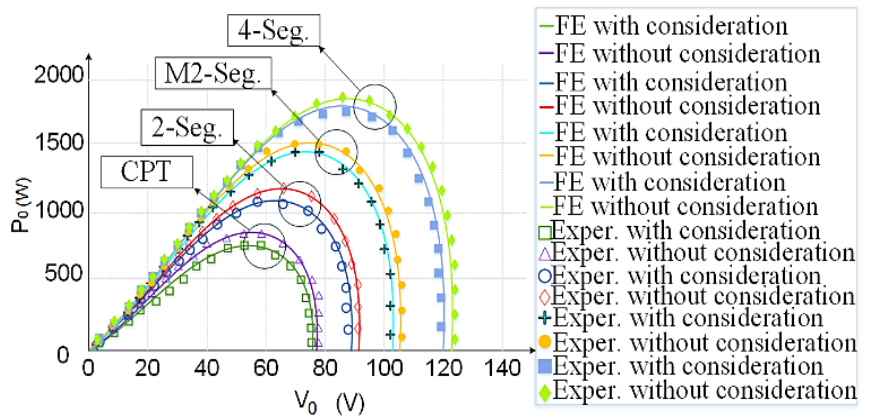

Fig. 11. Effect of gap segmentation consideration on the trend of $P_{0}$ to $V_{0}$

$1.1(\mathrm{~kW})$, respectively. Additionally, the Fig. 8, 9, and 10 have been summarized in Table II. This improvement of the HABObased models has been carried out due to a number of circumstances such as 
1) Higher flux linkage waveforms that are better suited to the studied geometries

2) More sinusoidal radial magnetic flux density

3) Fewer space and time harmonics

4) Increased fit of the sinusoidal back-EMF waveforms

TABLE I MAXIMUM POWER CALCULATION WITH PF=0.95

\begin{tabular}{l|llll}
\hline$M P$ & $C P T$ & 2 -Seg. & $M-2$-Seg. & 4-Seg. \\
\hline$I_{L}(\mathrm{~A})$ & 0.96 & 1.037 & 1.038 & 1.107 \\
$I_{\text {rec }}(\mathrm{A})$ & 0.60 & 0.61 & 0.61 & 0.63 \\
$P_{a c}(\mathrm{kVA})$ & 1.16 & 1.37 & 1.44 & 1.66 \\
$P_{0}(\mathrm{~kW})$ & 1.17 & 1.65 & 1.67 & 1.93 \\
\hline
\end{tabular}

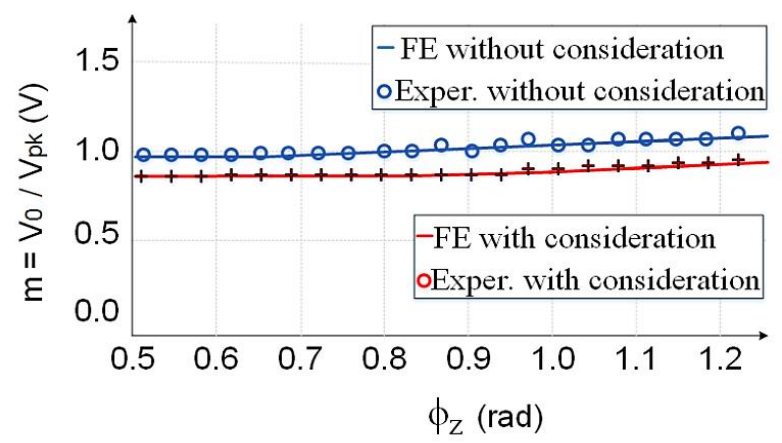

Fig. 12. The theoretical and experimental trends of $P_{0}$ versus $\varphi_{z}$ with and without gap consideration for proposed 4-segmented HABO model

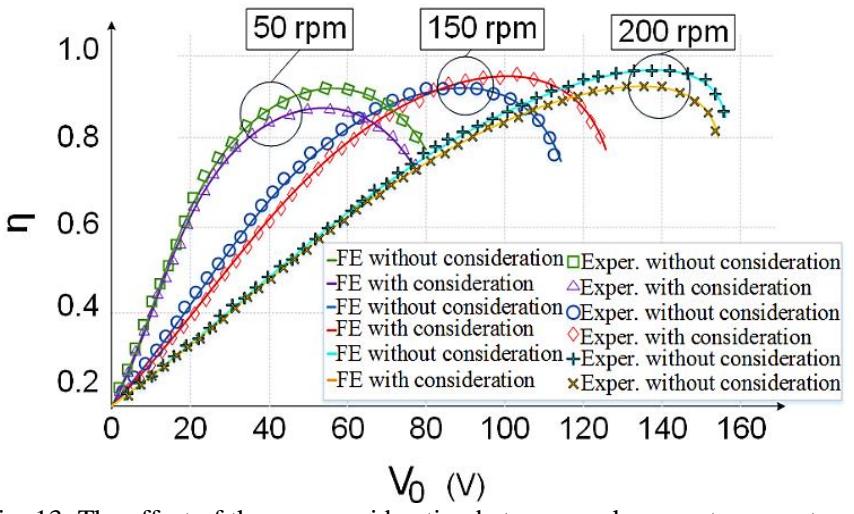

Fig. 13. The effect of the gap consideration between each magnet segment on the efficiency (rectifier load included) and output voltage

Fig. 11, presents how much both numerically and experimentally the gap consideration between each magnet segment will have an impact on output DC power $\left(P_{0}\right)$ tendency as a function of output voltage $\left(V_{0}=V_{D C}\right)$ at the rated speed. The "green curve" and "purple curve" show the theoretical trend for the CPT with and without the gap consideration, respectively. In addition, the "green square" and "purple triangle" demonstrate the experimental verifications on the related curves. The "blue" and "red" curves are showing the 2-segmented HABO with and without gap consideration. The "turquoise" and "orange" curves and points refer to the theoretical and experimental verification with and without gap consideration for modified-2-segmented HABO. The "cerulean blue" and "light green" curves and points indicate the tendency with and without gap consideration for the 4-segmented $\mathrm{HABO}$, where the points illustrate the experimental values.

According to [8], the two key design parameters $m$ and $\varphi_{z}$ have been analyzed under maximum power tracking (MP) operation, where through a given $\varphi_{z}$, an approximation of $m_{M P}\left(\varphi_{z}\right)$ can be given for applicable evaluations as

$$
m_{M P}\left(\varphi_{z}\right) \cong\left\{\begin{array}{c}
0.83 \rightarrow \text { if }: 0<\varphi_{z} \leq 0.72(\mathrm{rad}) \\
0.289 \varphi_{Z}^{2}-310 \varphi_{z}+0.769 \rightarrow \text { otherwise }
\end{array}\right.
$$

Therefore Fig. 12 represents the theoretical and experimental tendencies of $P_{0}$ versus $\varphi_{z}$ with and without gap consideration for the proposed 4-segment HABO model, in which the "red" and "blue" curves have indicated the model with and without gap consideration. Additionally, the related points showed the experimental values.

Fig. 13 is presented to verify the trend of the calculated (curves) and experimental (points) efficiency versus output DC power as a function of speed with and without consideration of gap. At 50 $(\mathrm{rpm})$, the maximum efficiency and voltage values are $91 \%$, and 58 (v). Also, the maximum reachable efficiency value at 150 (rpm), and 100 (v) has been reported as $98 \%$. At 200 (rpm), the efficiency value of 0.985 is greater, but not preferable due to the intense increase in voltage observed in this study. It should also be mentioned that the small discrepancy between numerical and experimental values is due to the assumption of neglecting the eddy currents in the rotor core and magnets induced via the stator current harmonics.

TABLE III CO-ENERGY CALCULATION [JOULES]

\begin{tabular}{c|ccccc}
\hline Models & Initial & 2-Seg & $\begin{array}{c}\text { M_2- } \\
\text { Seg. }\end{array}$ & 4-Seg. & Polar ani. \\
\hline Analytical & 91.1 & 145.9 & 159.4 & 143.4 & 144.7 \\
FEA & 90.33 & 145.2 & 158.8 & 142.8 & 143.66 \\
\hline
\end{tabular}

TABLE IV THE SUMMARIZED CALCULATED LOAD RESUlTS

\begin{tabular}{c|ccccc}
\hline Model & $I_{r m s}[A]$ & $V_{d c}[v]$ & $P_{s}[V A]$ & $T_{m}[N m]$ & $P_{0}[k W]$ \\
\hline CPT & 0.6 & 594 & 1174 & -72.6 & 1.1 \\
2-Sg & 0.7 & 644 & 1377 & -83.7 & 1.3 \\
M2Sg & 0.68 & 661 & 1447 & -89.3 & 1.39 \\
4Seg & 0.71 & 709 & 1675 & -103 & 1.6 \\
\hline
\end{tabular}

TABLE V GEOMETRY MACHINE DESIGN

\begin{tabular}{c|cc}
\hline Variable & \multicolumn{2}{|c}{ Linear current density fixed } \\
\hline $\mathrm{l}_{\mathrm{s}}$ & 100 & $\mathrm{~mm}$ \\
$D_{s h}$ & 50 & $\mathrm{~mm}$ \\
$D_{s e}$ & 410 & $\mathrm{~mm}$ \\
$\delta_{\mathrm{g}}$ & 0.6 & $\mathrm{~mm}$ \\
$\mathrm{~S}_{\mathrm{w}}$ & 15 & $\mathrm{~mm}$ \\
$S_{d}$ & 50 & $\mathrm{~mm}$ \\
$Q_{s}$ & 36 & \\
$\alpha_{p}$ & 0.55 & \\
$2 P$ & 40 & \\
$m$ & 3 & \\
\hline
\end{tabular}

Similarly, the Table. III shows the produced co-energy [13] of the different models that is directly related to the EM output power of the SPMSGs. It can thus be observed that having more magnetic parts does not necessarily increase the co-energy production. For example, the polar anisotropic model neglects the segmentation and gap considerations which can be compared with other HABObased models and is approximately equal in production. Additionally, the Table. IV illustrates the diode rectifier load average results in comparison with all the studied models. Table. $\mathrm{V}$ represents the initial input data which was involved in the first stage of the methodology (Fig.5). Here, $\mathrm{l}_{\mathrm{s}}, D_{s h}, L_{m}, \delta_{\mathrm{g}}, S_{d}, Q_{s}, \alpha_{p}$, $2 P$, and $m$ are active stack stator length, shaft diameter, rotor diameter, thickness of permanent magnet, air-gap length, effective slot-depth, total number of the stator slots, the relative pole-arc coefficient, number of poles, and stator phase number, 
respectively.

\section{Conclusion}

Determining the effectiveness of the gap consideration for the CPT and HABOs-based permanent magnet synchronous generator was the objective of this study. The exterior rotor models feeding a three-phase diode rectifier load. A coupled field-circuit study via 2-D FEA was examined to evaluate the outcomes by considering the effect of eddy-currents. The coupled load capabilities were considered and analyzed both through the instantaneous value of field and circuit variables as well as the equivalent DQ-axis model.

In this study, the computed results of the 4-segmented HABO model with $1.6(\mathrm{~kW})$ using MP, and usable DC voltage of 709 (v) was presented. The analysis was performed with accurately calculated power factors of the studied models at 0.95 which demonstrates that the width/pole pitch ratio and the length/pole pitch ratio can be considered equal for the CPT and HABO-based models. However, numerous different load results could be reported. It should be mentioned that the produced co-energy through the CPT has not been compared with the HABO-based models due to different magnet volumes. The modified-2segmente co-energy production with $158.8(\mathrm{~J})$, in comparison with the 4-segmented HABO model with $143.66(\mathrm{~J})$ has achieved a significantly greater value. Thus, the load performance is impacted by the gap consideration, the magnet's volume usage, and how the flux linkages effect the geometry of the model.

Additionally, the numerical results have been verified through experimental values with less than $3 \%$ error, and the major output parameters have been comprehensively compared with and without gap consideration. Depending on the model, the resulting error varied between " 5 to $8 \%$ ". Thus, the proposed 4 -segmented HABO model with $5.93 \%$ error in output values without gap consideration was selected.

\section{References}

[1] Y. Feng, X. H. Yu, and F. L. Han, "High-order terminal sliding-mode observer for parameter estimation of a permanent-magnet synchronous motor," IEEE Trans. Ind. Electron., vol. 60, no. 10, pp. 4272-4280, Oct. 2013.

[2] W. M. Lin, T. J. Su, and R. C. Wu, "Parameter identification of induction machine with a starting no-load low-voltage test," IEEE Trans. Ind. Electron., vol. 59, no. 1, pp. 352-360, Jan. 2012.

[3] R. Babau, I. Boldea, T. J. E. Miller, and N. Muntean, "Complete parameter identification of large induction machines from no-load acceleration-deceleration tests," IEEE Trans. Ind. Electron., vol. 54, no. 4, pp. 1962-1972, Aug. 2007.

[4] N. J. Kim, H. S. Moon, and D. S. Hyun, "Inertia identification for the speed observer of the low speed control of induction machines," IEEE Trans. Ind. Appl., vol. 32, no. 6, pp. 1371-1379, Sep./Oct. 1996.

[5] S. H. Li and Z. G. Liu, "Adaptive speed control for permanent-magnet synchronous motor system with variations of load inertia," IEEE Trans. Ind. Electron., vol. 56, no. 8, pp. 3050-3059, Aug. 2009.

[6] F. J. Lin and H. M. Su, “A high-performance induction motor drive with on-line rotor time-constant estimation," IEEE Trans. Energy Convers., vol. 12, no. 4, pp. 297-303, Dec. 1997.

[7] F. Andoh, "Moment of inertia identification using the time average of the product of torque reference input and motor position," IEEE Trans. Power Electron., vol. 22, no. 6, pp. 2534-2542, Nov. 2007.

[8] Matteo F. Iacchetti, Giovanni Maria Foglia, Antonino Di Gerlando, and Andrew J. Forsyth, "Analytical Evaluation of Surface-Mounted PMSG Performances Connected to a Diode Rectifier," IEEE Trans. Energy Convers., vol. 30, no. 4, pp. 1367-1374, Dec. 2015

[9] Ma Weiming, Hu An, Liu Dezhi, and Zhang Gaifan "Stability of a Synchronous Generator with Diode-Bridge Rectifier and Back-EMF Load," IEEE Trans. Energy Convers., vol. 15, no. 4, pp. 458-463, Dec. 2000

[10] Weimin Wang, K. W. E. Cheng, K. Ding, and L. C. Meng, "A Novel Approach to the Analysis of the Axial-Flux Permanent-Magnet
Generator With Coreless Stator Supplying a Rectifier Load," IEEE Trans. Magnetics., vol. 47, no. 10, pp. 2391-2394, Dec. 2011.

[11] Pedram Asef, R. B. Perpina, and M. R. Barzegaran "Electromagneticbased Evaluation of Different Halbach-Array topologies on the Exterior Rotor PM Synchronous Machines." IEEE Trans. Ind. Electron., status: under view, Dec. 2016.

[12] W. Will, "Introduction to Understandable Physics," Chapter book, Vol. 1- Mechanics. AuthorHouse. p. 10.10. ISBN 1449063330, 2010.

[13] J. R. Hendershot, and T. J. E. Miller, "Design of Brushless Permanent Magnet Machines." Oxford University Press, pp.185-465, 2010.

[14] Tze-Fun Chan, W. Wang, and L. Lei Lai, "Design of Brushless Permanent Magnet Machines." IEEE International Electric Machines and Drives Conference, pp. 1600-1604, 2009.

[15] M. C. McCalla, Fundamentals of Computer-Aided Circuit Simulation, Kluwer Academic, (1988)

[16] W. N. Fu, and S. L. Ho, "Enhanced Nonlinear Algorithm for the Transient Analysis of Magnetic Field and Electric Circuit Coupled Problems," IEEE Trans. Magnetics., vol. 45, no. 2, pp. 701-705, Feb. 2009.

[17] A. Di Gerlando, G. M. Foglia, M. F. Iacchetti, and R. Perini, "Comprehensive steady-state analytical model of a three-phase diode rectifier connected to a constant DC voltage source," IET Power Electron., vol. 6, no. 9, pp. 1927-1938, Nov. 2013.

[18] J. Pyrhonen, T. Jokinen, and V. Hrabvocova, Design of Rotating Electrical Machines. Hoboken, NJ USA: Wiley, 2008, pp. 259-261.

[19] T. F. Chan, L. L. Lai and L. T. Yan, "Analysis of a stand-alone permanent-magnet synchronous generator using a time-stepping coupled field-circuit method," IEE Proc.-Electric Power Applications. vol. 152, no. 6, pp. 1459-1467, Nov. 4, 2005.

[20] Xiaoxu Zhang, Xiao Liu, Jinglin Liu, and Zhe Chen, "Analytical Investigation on the Power Factor of a Flux-Modulated PermanentMagnet Synchronous Machine," IEEE Trans. Magnetics., vol. 51, no. 11, pp. 1-3, Feb. 2015.

[21] M. Vukotic, and D. Miljavec, "Design of a permanent-magnet fluxmodulated machine with a high torque density and high power factor," IET Electric Power Applications, Volume: 10, Issue: 1, DOI: 10.1049/iet-epa.2015.0143, 201
Click here to remove instruction text.. 